\title{
Reinemann, Carsten; Stanyer, James; Aalberg, Toril; Esser, Frank; de Vreese, Claes H. (eds.): Communicating Populism. Comparing Actor Perceptions, Media Coverage, and Effects on Citizens in Europe
}

\author{
New York \& London: Routledge. 279 Seiten. Preis: $£ 120$
}

\section{Klaus Kamps}

Online publiziert: 17. März 2020

(C) Der/die Autor(en) 2020

Communicating Populism ist ein internationales Gemeinschaftswerk und versammelt eine Reihe größerer Forschungsarbeiten zur Analyse von Populismus aus vergleichender Perspektive. Konkret umfasst der Band vier gut dokumentierte Studien: zwei Befragungen von Journalisten und Politikern, eine Inhaltsanalyse und eine Experimentalstudie auf der Basis national repräsentativer Online-Umfragen - im großen Maßstab und für bis zu 15 Länder. Innerhalb der rasant wachsenden sozialwissenschaftlichen Literatur zu Populismus und populistischer Kommunikation sticht das Werk mit diesem komparativen Ansatz heraus. Es gibt wichtige Hinweise für Ansatzpunkte in der Forschung und dürfte dementsprechend auch richtungsweisend sein für künftige Arbeiten.

Ausgangspunkt des Bandes sind zwei zentrale Überlegungen. Erstens: die Sozialwissenschaften haben den Faktor Kommunikation an und für sich als wichtiges Element zur Erklärung von Populismus (und seinen Folgen) zwar erkannt und benannt, in den Studien hingegen nur unzureichend berücksichtigt. Sozialforschung, die z.B. die Beweggründe von Wählerinnen und Wählern populistischer Parteien untersucht, impliziert zwar meist, dass Medien und Journalismus an deren Meinungsbildung beteiligt sind (und sein müssten), expliziert das indes eher selten, etwa aus journalistischer Perspektive. Zweitens wird argumentiert, dass die Populismusforschung von einer komparativen Analyse erheblich profitieren dürfte, nicht zuletzt, weil Populismus in höchst unterschiedlichen Kontexten zu finden ist, sich ausnehmend variantenreich präsentiert und, näher betrachtet, ein geradezu hyperkomplexes Phänomen darstellt. Dabei hat die Populismusforschung schon länger Hinweise gegeben auf die vielfältigen systemischen und nationalen Bedingungen, die den Erfolg oder Misserfolg populistischer Parteien oder Bewegungen zumin-

Dr. K. Kamps (凶)

Hochschule der Medien, Nobelstraße 10, 70569 Stuttgart, Deutschland

E-Mail: kamps@hdm-stuttgart.de 
dest annäherungsweise erklären können. Zum besseren Verständnis drängt sich ein komparativer Ansatz nachgerade auf.

Vor diesem Hintergrund konzentriert sich Communicating Populism auf Medien und Kommunikatoren und Rezeption. Der Anspruch ist dabei nicht, den Wert komparativer Sozialforschung in aller Breite darzulegen. Vielmehr werden die vorhandenen Studien durch ein stimmiges komparatives Forschungsprogramm unterfüttert. Das basiert auf einem Makro-, Meso-, Mikromodell und wird konsequent umgesetzt. Konsequenz ist überhaupt eine der Stärken des Buches: es ist kompakt und sehr stringent in der Leserführung und den Argumentationslinien. Es ist im besten Sinne handlich: Zugänglich und aktuell.

Wer sich für Befunde im Einzelnen interessiert, dem sei natürlich ein genauerer Blick in den Band empfohlen. (Der selbst mit einem zusammenfassenden Beitrag schließt.) An dieser Stelle sollen einige Linien einen ersten Eindruck festhalten. Auffällig ist eine Art Generalbotschaft, die auf allen vier Einzelstudien basiert: Dass nämlich keine einfachen Zusammenhänge oder gar Muster populistischer Kommunikation im Ländervergleich erkennbar sind. Das sei nachgerade ein auffälliger Gegensatz zu einem naiven Verständnis von Populismus als schlichtem Politikangebot, das meist auf eine Kommunikationsform rekurriere, die einfache, klar bestimmbare Mechanismen nutze. So gibt es einerseits zwar Gemeinsamkeiten - wie z.B. auf der Makroebene die Bedeutung von ökonomischen Entwicklungen und gesellschaftlichen Krisen wie etwa Flüchtlingskrise und jahrelange Auseinandersetzung um Migration und Integration. Aber wie genau sich die Dynamik populistischer Kommunikation im nationalen Konflikt dann entfaltet, ist von systemischen Besonderheiten abhängig: von beispielsweise journalistischen Kulturen und Selbstverständnissen, Eigenarten des Mediensystems und wohl auch historischen Erfahrungen von Ländern und ihren politischen Eliten. Ähnliches gilt für die Rezeption (und den möglichen politischen Erfolg) von populistischen Inhalten und die Wahrnehmung von Populisten selbst. Kurz: der Band zeigt die Komplexität populistischer Kommunikation auf - und auch, dass sich einfache Antworten verbieten. Die Autoren gehen letztlich davon aus, dass für weitere Klärungen eine Erweiterung ihres $\mathrm{Zu}$ gangs sinnvoll ist und die Medien- und Informationssysteme breiter eingebunden werden müssen. Gemeint sind Schlüsselfaktoren der Social Media-Kommunikation, Nachrichtenrepertoires in modernen Informationswelten oder auch Unterhaltung, Satire oder stark emotionalisierende Politikangebote.

Im Schlussteil des Bandes findet sich schließlich ein Abschnitt „Implications“, der sich nicht den Beschränkungen des eigenen Forschungsdesigns widmet, sondern Hinweise gibt für den praktischen Umgang mit Populismus für Journalistinnen und Journalisten, für Politiker und Politikerinnen. Man mag von solchen Empfehlungen halten, was man will: hier sind sie hilfreich. Sie basieren auch nicht allein auf dem konkreten Forschungsprogramm (und ,entspringen“ gleichsam den immer doch beschränkten Befunden), sondern schließen die aktuelle Forschung insgesamt ein.

In der Gesamtschau also: ein mitunter sogar spannend zu lesendes Buch, das nicht nur für Sozialforscher interessant sein dürfte, die sich konkret mit Populismus auseinandersetzen, sondern auch für Lehrende der Sozialwissenschaften und Personen, die in außerakademischen Zusammenhängen mit Populismus konfrontiert werden. 
Funding Open Access funding provided by Projekt DEAL.

Open Access Dieser Artikel wird unter der Creative Commons Namensnennung 4.0 International Lizenz veröffentlicht, welche die Nutzung, Vervielfältigung, Bearbeitung, Verbreitung und Wiedergabe in jeglichem Medium und Format erlaubt, sofern Sie den/die ursprünglichen Autor(en) und die Quelle ordnungsgemäß nennen, einen Link zur Creative Commons Lizenz beifügen und angeben, ob Änderungen vorgenommen wurden.

Die in diesem Artikel enthaltenen Bilder und sonstiges Drittmaterial unterliegen ebenfalls der genannten Creative Commons Lizenz, sofern sich aus der Abbildungslegende nichts anderes ergibt. Sofern das betreffende Material nicht unter der genannten Creative Commons Lizenz steht und die betreffende Handlung nicht nach gesetzlichen Vorschriften erlaubt ist, ist für die oben aufgeführten Weiterverwendungen des Materials die Einwilligung des jeweiligen Rechteinhabers einzuholen.

Weitere Details zur Lizenz entnehmen Sie bitte der Lizenzinformation auf http://creativecommons.org/ licenses/by/4.0/deed.de.

Dr. Klaus Kamps ist Professor für Kommunikationswissenschaft an der Hochschule der Medien in Stuttgart. 